\title{
Membrane Potential and Electrostatics of Phospholipid Bilayers with Asymmetric Transmembrane Distribution of Anionic Lipids
}

\author{
Andrey A. Gurtovenko*,† and Ilpo Vattulainen ${ }^{\ddagger}, \S, \|$ \\ Computational Biophysics Laboratory, Institute of Pharmaceutical Innovation, University of Bradford, \\ Bradford, West Yorkshire, BD7 1DP, U.K., Institute of Physics, Tampere University of Technology, P.O. Box \\ 692, FI-33101 Tampere, Finland, Helsinki University of Technology, P.O. Box 1100, FI-02015 HUT, Finland, \\ and MEMPHYS - Center for Biomembrane Physics, University of Southern Denmark, Odense, Denmark
}

Received: January 9, 2008; In Final Form: February 11, 2008

\begin{abstract}
It is well-established that native plasma membranes are characterized by an asymmetric distribution of charged (anionic) lipids across the membrane. To clarify how the asymmetry can affect membrane electrostatics, we have performed extensive atomic-scale molecular dynamics simulations of asymmetric lipid membranes composed of zwitterionic (phosphatidylcholine (PC) or phosphatidylethanolamine (PE)) and anionic (phosphatidylserine (PS)) leaflets. It turns out that the asymmetry in transmembrane distribution of anionic lipids gives rise to a nonzero potential difference between the two sides of the membrane. This potential arises from the difference in surface charges of the two leaflets. The magnitude of the intrinsic membrane potential was found to be $238 \mathrm{mV}$ and $198 \mathrm{mV}$ for PS/PC and PS/PE membranes, respectively. Remarkably, this potential is of the same sign as the membrane potential in cells. Our findings, being in reasonable agreement with available experimental data, lend support to the idea that the transmembrane lipid asymmetry typical of most living cells contributes to the membrane potential.
\end{abstract}

\section{Introduction}

Asymmetry in distribution of lipids across cellular membranes is an inherent feature of most eukaryotic cells. ${ }^{1}$ In particular, it is well-established that the extracellular leaflets of biological plasma membranes are mostly composed of cholinephospholipids, such as phosphatidylcholine (PC) and sphingomyelin, while aminophospholipids, such as phosphatidylethanolamine (PE) and phosphatidylserine (PS), are predominantly located in the intracellular leaflets. ${ }^{2}$ The asymmetric transmembrane lipid distribution is known to be vital for many physicochemical properties and functions of biological membranes; these include membrane mechanical stability ${ }^{3}$ and programmed cell death. ${ }^{4}$

What is considerably less known is the effect of the transmembrane asymmetry on the electrostatic properties of cell membranes. Plasma membranes of living cells possess a nonzero potential across the membrane. This membrane potential is a key player in many membrane-mediated phenomena such as binding of charged proteins and drugs to membranes, conductance of ionic channels, insertion and orientation of integral membrane proteins, and transport processes across the membrane. ${ }^{5}$ Despite its major biological relevance, the origin of this potential is far from being well understood.

A generally accepted view is that the transmembrane potential difference arises from a charge imbalance of salt ions across the plasma membrane. ${ }^{6}$ Indeed, the concentrations of, e.g., sodium and potassium ions inside and outside the cell differ considerably. However, the nature of the membrane potential can be much more involved than the traditional view presented

\footnotetext{
* Corresponding author. E-mail: A.Gurtovenko@bradford.ac.uk

$\dagger$ University of Bradford.

$\doteqdot$ Tampere University of Technology.

$\S$ Helsinki University of Technology.

" University of Southern Denmark.
}

above. In particular, several experimental studies of asymmetric lipid membranes have supported the idea that an asymmetric distribution of lipid molecules across the membrane can also give rise to a nonzero electrostatic potential between the two membrane leaflets. ${ }^{7-9}$ A recent atomic-scale simulation study confirmed this view by showing that the transmembrane potential can be nonzero even in the absence of salt ions, provided that the lipid distribution is asymmetric. ${ }^{10}$

In general, one should distinguish three different contributions that can make the intrinsic membrane potential nonzero (in addition to the ionic charge imbalance across the membrane): (i) an asymmetric transmembrane distribution of zwitterionic (neutral) lipid molecules, (ii) an asymmetric distribution of charged (mainly anionic) lipids and their counterions over the two leaflets, and (iii) an effect of salt ions on the lipid membrane. All the situations are highly relevant to biological plasma membranes. The first step in the systematic study of the complex nature of the intrinsic membrane potential would be the consideration of the three contributions separately, thereby revealing the generic features of each of them.

(i) As far as zwitterionic lipids are concerned, they are known to be the major lipid species in cell membranes, the dominant lipid types being very different for the inner and outer leaflets. Remarkably, the transmembrane asymmetry of zwitterionic lipids turns out to be coupled to the intrinsic membrane potential. Dipole potential measurements of asymmetric membranes with leaflets composed of bacterial PE and 1,3-diolein (two lipids with different polar (neutral) head groups) revealed a nonzero intrinsic membrane potential. ${ }^{7}$ Recent atomistic simulations of an asymmetric PC/PE membrane allowed one to draw a similar conclusion. ${ }^{10}$ In both studies, a transmembrane potential difference of about $100 \mathrm{mV}$ was reported. Most likely, the origin of the nonzero membrane potential for asymmetric lipid 
membranes of that type results from a subtle difference in the dipole moments of the two zwitterionic leaflets.

(ii) The situation is more complicated when a membrane includes charged (anionic) lipids as well as their counterions. This case of an asymmetric charged membrane is most typical for living cells: PS lipids characterized under physiological conditions by a net negative charge are mainly located in the inner leaflets of cell membranes. What is more, the appearance of PS lipids in the outer leaflet (externalization) initiates cell clearance and eventually leads to cell death. ${ }^{11}$ Therefore, most animal cells restrict negatively charged PS lipids to the inner leaflets, leading to a pronounced asymmetrical distribution of lipid charges across the membrane. Such a difference in surface charges on the two sides of the membrane apparently can induce a nonzero transmembrane potential difference; a few experimental studies ${ }^{8,9}$ have provided some evidence that this is possibly the case.

(iii) As far as salt ions are concerned, they are known to interact with lipid molecules and therefore affect the structural and dynamic properties of cell membranes. ${ }^{12-15}$ Recent atomicscale computational studies demonstrated that salt ions are able to influence the membrane electrostatics. ${ }^{12,16-18}$ Furthermore, the effect of monovalent salt was found to differ considerably for the inner and outer leaflets of plasma membranes. ${ }^{19}$ However, the cumulative effect of salt ions and transmembrane lipid asymmetry on the membrane electrostatics is still to be understood in detail. Our preliminary studies show that these two factors can even cancel each other to a certain extent [Gurtovenko, A. A.; Vattulainen, I., 2007, unpublished work].

Considering the many factors that have a role to play in the membrane potential, it is evident that detailed atomistic simulations could provide a great deal of insight into understanding the nature and origin of the potential. Rather surprisingly, though, there is currently only one study that has elaborated on this matter. ${ }^{10}$ In the present work, we discuss this issue further by considering the contribution of the asymmetric transmembrane distribution of charged (anionic) lipids to the intrinsic membrane potential (item (ii) in the above list), as the role of this type of lipid asymmetry is still not well understood. To elucidate this problem, we have performed atomic-scale molecular dynamics (MD) simulations of asymmetric membranes composed of zwitterionic and anionic lipid monolayers. Two different bilayer systems were considered, namely asymmetric $\mathrm{PC} \mathrm{PS}^{-}$and $\mathrm{PE} / \mathrm{PS}^{-}$membranes. Although the outer leaflets of most plasma membranes consist of not more than $20-30 \%$ of PS lipids, ${ }^{2}$ here we chose to consider one of the two leaflets to be formed exclusively of anionic lipids to make the effect of the asymmetry most pronounced.

Overall, our simulations provide compelling evidence that lipid membranes with an asymmetric transmembrane distribution of anionic lipids can be characterized by a nonzero potential difference between two surfaces of the membrane, lending support to the idea that the membrane asymmetry and the intrinsic electrostatic potential in cell membranes are coupled.

\section{Methods}

We have performed atomic-scale MD simulations of two asymmetric lipid membranes composed of zwitterionic (palmitoyl-oleoyl-phosphatidylcholine (POPC), or palmitoyl-oleoylphosphatidylethanolamine (POPE)) and anionic (palmitoyloleoyl-phosphatidylserine (POPS)) single-component monolayers. Force-field parameters for POPC and POPE lipids were taken from the united atom force-field of Berger et al.; ${ }^{20} \mathrm{PE}$ head groups were described following the POPE model of Tieleman and Berendsen. ${ }^{21}$ Force-field parameters for POPS lipids were taken from ref 22. Water was modeled using the simple point charge (SPC) model. ${ }^{23}$ As counterions of anionic POPS lipids, we used sodium ions for which the set of parameters supplied within the GROMACS force-field was employed. ${ }^{24}$ The Lennard-Jones interactions were cut off at $1 \mathrm{~nm}$. The particle-mesh Ewald (PME) method ${ }^{25,26}$ was used for electrostatic interactions. The simulations were performed in the $N p T$ ensemble at physiological temperature $(T=310 \mathrm{~K})$ and at a pressure set to 1 bar; the Berendsen scheme was employed for both temperature and pressure. ${ }^{27}$ The periodic boundary conditions were applied in all three dimensions. The time step used was $2 \mathrm{fs}$.

Simulations of asymmetric lipid membranes were set up and performed in three steps:

(i) First, single-component symmetric bilayers composed of 128 POPC, POPE, and POPS lipids and around 5000 water molecules were simulated for a period of $100 \mathrm{~ns}$. On the basis of these simulations, the information regarding the equilibrium values for the area per POPC, POPE, and POPS lipids were extracted. For a POPC bilayer, the area per lipid was found to be $0.65 \mathrm{~nm}^{2}$, being in very good agreement with available experimental $^{28-31}$ and computational studies. ${ }^{12,32,33}$ For the area per lipid of single-component POPE and POPS bilayers, we found values of 0.52 and $0.535 \mathrm{~nm}^{2}$, respectively. Again, these values are in line with reported $\mathrm{MD}$ studies of $\mathrm{PE}^{32-34}$ and PS $^{22,35,36}$ membranes under similar conditions. On the experimental side, a value of $0.56 \mathrm{~nm}^{2}$ was found for the area per lipid for a POPE bilayer. ${ }^{37}$ The experimental data for PS lipid bilayers can scatter considerably, the reported values being in the range from 0.45 to $0.55 \mathrm{~nm}^{2} .{ }^{38,39}$ Therefore, the area per lipid for all the simulated bilayer systems is proved to be realistic, validating thereby the molecular model employed in this study.

(ii) Using the data for the area per lipid, two asymmetric membranes, PS/PC and PS/PE, were formed by adjoining POPS monolayers with POPE and POPC monolayers. The number of lipids in the two leaflets of the two asymmetric membranes was adjusted such that the average area per lipid in each leaflet reproduced closely the average area per lipid in corresponding single-component bilayers. This led us to an asymmetric PS/ PC membrane with 64 lipids in the POPS leaflet and 53 lipids in the POPC leaflet, and to a PS/PE membrane with 62 lipids in the POPS leaflet and 64 lipids in the POPE leaflet. The approach used here ensures that the spontaneous curvature of the bilayer corresponds to a flat membrane as in giant unilamellar vesicles.

(iii) Because of periodic boundary conditions, sodium ions needed to neutralize anionic PS lipids can easily appear on the zwitterionic side of an asymmetric membrane and get bound to its carbonyl region. ${ }^{12,17,18}$ To overcome this undesirable periodicity-related artifact, a double bilayer setup (i.e., two lipid bilayers in a simulation box) was employed (see Figure 1). Such a setup provides an independent control over the electrolyte composition of two aqueous compartments separated by bilayers and was successfully used in a number of preceding computational studies..$^{18,40-45}$ For the purposes of the present study, the double bilayer system was built up from two asymmetric membranes such that anionic POPS lipids were adjusted to the same water compartment; a proper number of $\mathrm{Na}^{+}$ions were then added randomly into that compartment to keep the system electroneutral (see Figure 1). In the resulting double bilayer system sodium ions do not have access to the zwitterionic leaflets. 


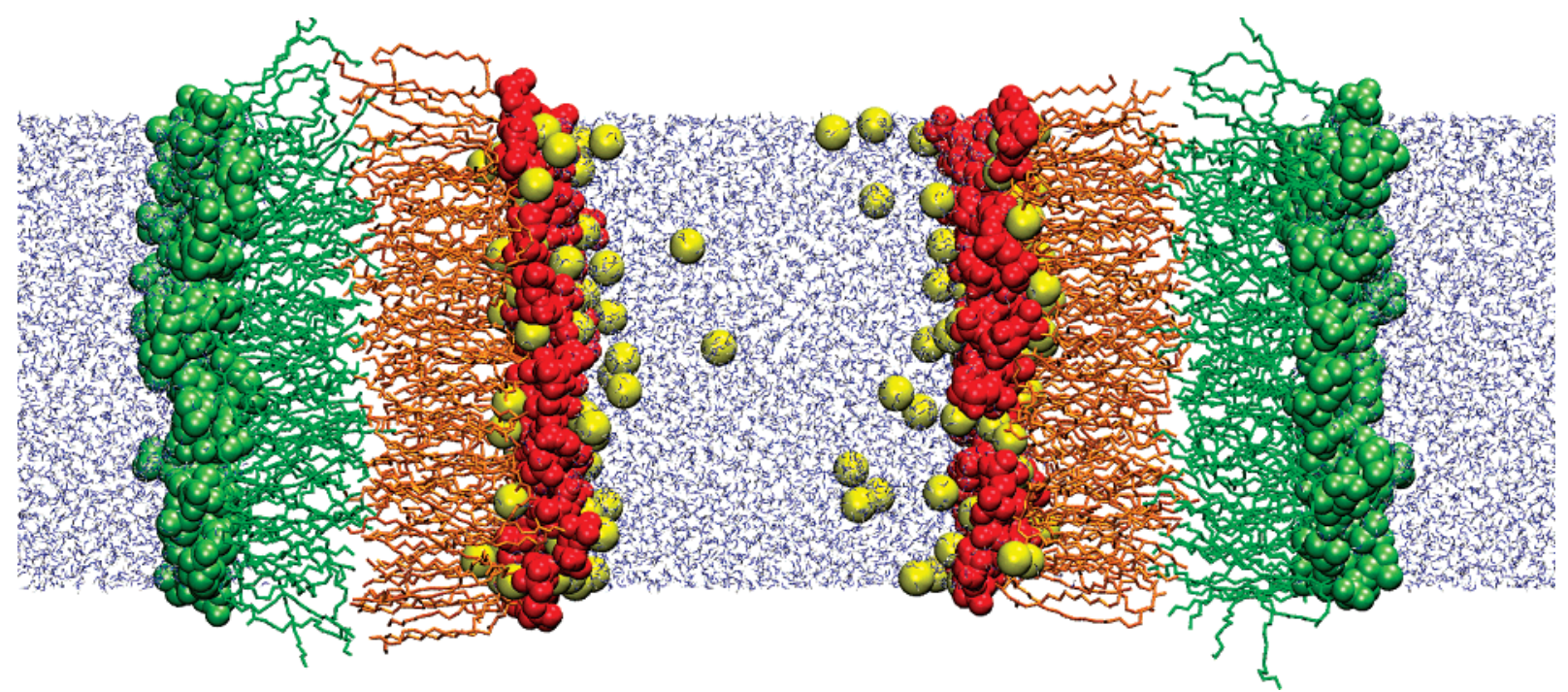

Figure 1. A snapshot of a double PC/PS ${ }^{-}$bilayer system in aqueous solution with $\mathrm{Na}^{+}$counterions after $100 \mathrm{~ns}$ of MD simulations. Shown are POPC lipids (head groups in lime, and acyl chains in green), POPS lipids (head groups in red, and acyl chains in orange), water (blue), and sodium ions (yellow).

Overall, a typical simulation system consists of two asymmetric lipid membranes (PS/PE or PS/PC membranes plus $\mathrm{Na}^{+}$ counterions) and from 10600 to 11500 water molecules, amounting to $\sim 45500$ and 47200 atoms for PS/PE or PS/PC membranes, respectively. Both asymmetric bilayer systems were simulated over a period of 100 ns. All simulations were performed using the GROMACS suite. ${ }^{24}$

In our previous study for zwitterionic lipids, we found that the membrane potential in an asymmetric membrane can deviate from zero even in the absence of salt. ${ }^{10}$ This implies that the potential in the vicinity of the two opposite membrane-water interfaces is different, and one is tempted to ask whether this plays any role in simulations with a single lipid bilayer surrounded by the water phase. In ref 10 , the results were confirmed by considering different boundary conditions for the single bilayer studied (using the slab geometry ${ }^{46}$ in addition to the commonly employed periodic boundary conditions). Later, we complemented those tests by repeating the simulations with the double bilayer setup and found that the results and conclusions do not change (unpublished data). Here, for the sake of certainty, we carry out all simulations employing the double bilayer scheme (see Figure 1).

\section{Results and Discussion}

A. Equilibration. System equilibration is of main concern in atomic-scale membrane simulations. This is particularly the case for membranes under the influence of ions, since the slowest relaxation process in such systems is associated with ion binding to the lipid/water interface. ${ }^{12,18,22}$ Therefore, along with the area per lipid, we monitor the time evolution of coordination numbers of $\mathrm{Na}$ ions with carbonyl and carboxylate oxygens of POPS lipids. It turns out that both the membrane area and the cation binding require around 30-35 ns for equilibration (data not shown). Correspondingly, only the part of MD trajectories over the last $60 \mathrm{~ns}$ (out of $100 \mathrm{~ns}$ ) was used for analysis.

B. Asymmetric PS/PC Membrane. An asymmetric membrane built from POPC and POPS leaflets can be considered as a rough model for plasma membranes: zwitterionic PC lipids are dominant in the outer leaflet, while most anionic PS lipids are localized in the inner monolayer. As mentioned in the Introduction, we intentionally enhance the effects of asymmetry by considering one of the two leaflets to be composed of anionic PS lipids only (inner leaflets of most plasma membranes contain usually no more than $30 \mathrm{~mol} \%$ of anionic lipids). A more elaborate study for realistic mixtures of many-component cellular membranes will be discussed elsewhere.

The component-wise density profiles of the asymmetric PS/ PC membrane are shown in Figure 2 (top). The difference between POPC and POPS lipids is essentially twofold and lies in the nature of their head groups. First, POPS head groups possess a net negative charge under physiological conditions, while zwitterionic POPC lipids are characterized by polar but neutral head groups. Second, POPS lipids have a primary amine in their head group region, thus POPS is capable of the formation of both intra- and intermolecular hydrogen bonds. In practice, the inter-lipid hydrogen bonding is so strong that it leads to a considerably more densely packed structure of a POPS monolayer as compared to its POPC counterpart, despite the electrostatic repulsion between charged POPS head groups. ${ }^{47}$

The more densely packed nature of the POPS leaflet is clearly seen from the peak heights of the lipid density profiles on the two sides of a membrane (see Figure 2 (top)). The average orientation of PN vectors (from phosphorus to nitrogen in the head group) with respect to the membrane normal on the two sides of the membrane was found to be similar: $74^{\circ}$ for POPS and $78^{\circ}$ for POPC. However, the structure of the water/lipid interface is more complex in the POPS case, as negatively charged PS head groups have to be accommodated in a rather narrow interface region. Furthermore, one observes a tight binding of sodium ions to the POPS leaflet interface. Remarkably, the density profile of sodium ions has two distinguished peaks (see Figure 2 (top)), indicating that $\mathrm{Na}^{+}$ions are able to bind to two distinct sites in the lipid/water interface, namely, to the carbonyl and carboxylate regions. A detailed analysis of corresponding coordination numbers shows that sodium ions mainly bind to the carbonyl oxygens located rather deep in the interface. $^{22}$

All the above features of POPC and POPS leaflets are translated into the partial charge densities, which are crucial for the electrostatic membrane properties (see Figure 2 (middle)). On the POPC side of the membrane, water molecules readily permeate into the lipid/water interface and reorient themselves to compensate for charges of phosphate and choline groups of 

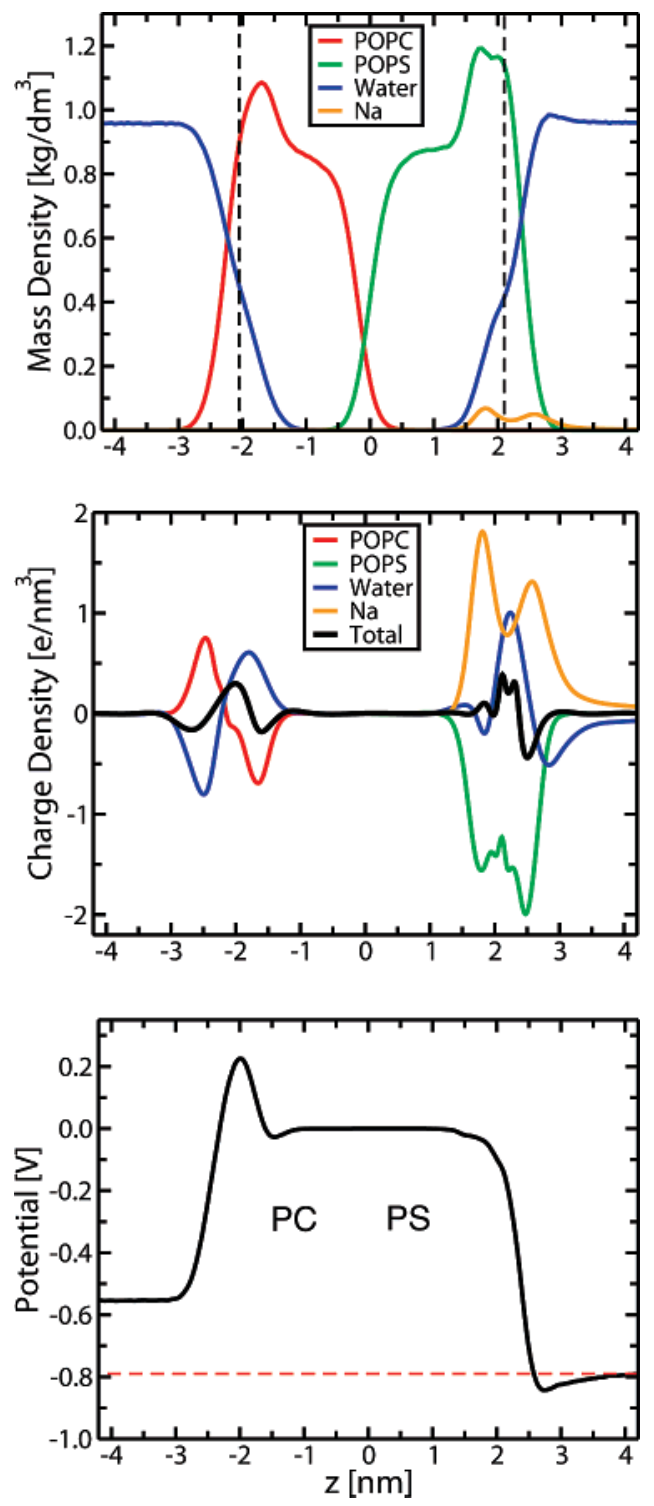

Figure 2. (top) Component-wise mass density profiles of an asymmetric POPS/POPC membrane as a function of distance $z$ from the center of mass of the membrane. All profiles are averaged over two membranes in a simulation box. The average positions of phosphate groups on both sides of the membrane (which define the membrane thickness) are shown by dashed lines. (middle) Charge density profiles of a POPS/POPC membrane. To reduce noise in the data, the charge densities shown here were fitted to splines. ${ }^{58}$ (bottom) Electrostatic potential across a POPS/POPC membrane. The potential is chosen to be zero at the center of mass of the membrane.

POPC lipids. This leads to a rather smooth profile for the total charge density. In contrast, deep water permeation into the POPS leaflet is considerably hindered by the densely packed anionic PS head groups. In turn, sodium ions penetrate much deeper into the leaflet compared to water and are mainly responsible for the compensation of a net negative charge of PS head groups (see Figure 2 (middle)); the charge density profile of $\mathrm{Na}^{+}$ions again demonstrates two distinguished maxima. Water also contributes to the compensation of the charges of POPS lipids, the reorientation of water molecules being more pronounced inside the PS leaflet compared to the POPC leaflet. As a result, the total charge density profile of the POPS leaflet has a more rugged shape as compared to that for the POPC leaflet, reflecting a more complex structure of the water/POPS interface. Overall, we observe a prominent asymmetry in charge distribution across the POPS/POPC asymmetric membrane.
The electrostatic potential across an asymmetric PS/PC membrane was computed from the Poisson equation by twice integrating over the charge densities measured from MD simulations (see Figure 2 (bottom)). As evident, the resulting electrostatic potential of the PS/PC membrane turns out to be asymmetric with respect to the membrane center: there is a nonzero potential difference of $238 \pm 19 \mathrm{mV}$ between the two surfaces of the membrane. It is important to emphasize that this membrane potential is not essentially related to the ionic charge imbalance across the membrane. Instead, there are two factors that contribute to the potential: the difference in the surface charges of anionic and zwitterionic leaflets of the PS/PC membrane, and the contribution due to counterions. Figure 2 (middle) depicts the charge densities of POPS, water, and counterions. Comparing these with the total charge density reveals that the qualitative form of the total charge density follows the contribution due to POPS. The role of $\mathrm{Na}^{+}$ counterions is of opposite nature but weaker than the contribution arising from PS head groups. Nonetheless, it is evident that counterions and charged lipid head groups compete with each other, and the total membrane potential results from their complex interplay. In more realistic situations with physiological salt conditions, it is likely that the same competition takes place.

The overall picture here is reminiscent of the situation observed in MD simulations of a PC membrane that separated two water reservoirs with and without salt. ${ }^{18}$ In that study, binding of sodium ions took place only in the membrane leaflet adjoined to salt, so that one of the leaflets effectively became positively charged, while the other one stayed neutral (zwitterionic). Such a salt-induced asymmetry of a lipid bilayer resulted in a nonzero potential difference between the two membrane sides. ${ }^{18,43}$

The different composition in the two leaflets also gives rise to a prominent asymmetry in the shape of the electrostatic potential across the POPS and POPC leaflets. In particular, one can notice the absence of a hump (typical in PC bilayers) close to the water/lipid interface for the potential on the POPS side of the membrane (see Figure 2 (bottom)). The origin of the hump observed in PC membranes lies in a subtle imbalance between the orientation of water molecules and PC lipid head groups. In PS membranes, such an imbalance is most likely suppressed by the strong electrostatic interactions between anionic head groups and counterions. We also find that the shape of the electrostatic potential and the value of the potential difference across PS and PC monolayers follow closely those for singlecomponent symmetric POPC and POPS bilayers and are in line with previous computational results. ${ }^{10,12,36}$

Importantly, the observed intrinsic potential of the membrane in the inside of the "cell" turns out to be negative with respect to the outside, provided that the POPC monolayer of the asymmetric PS/PC membrane is considered to reflect the outer leaflet of a plasma membrane. Thus, the intrinsic potential due to asymmetry in the distribution of charged lipids across the membrane has the same sign as the transmembrane potential in plasma membranes. As experimental measurements for PS/PC membranes are not available, a direct comparison of the magnitude of the transmembrane potential difference $(238 \mathrm{mV})$ with experiment is not feasible. We note, however, that in plasma membranes of living cells, one can expect the corresponding potential difference to be considerably smaller than the reported value as only $20-30 \mathrm{~mol} \%$ (and not $100 \%$ as in the simulations) of all lipids in the inner leaflet are anionic.

It would be interesting to compare our results with a recent MD study of asymmetric lipid membranes composed of a PC 

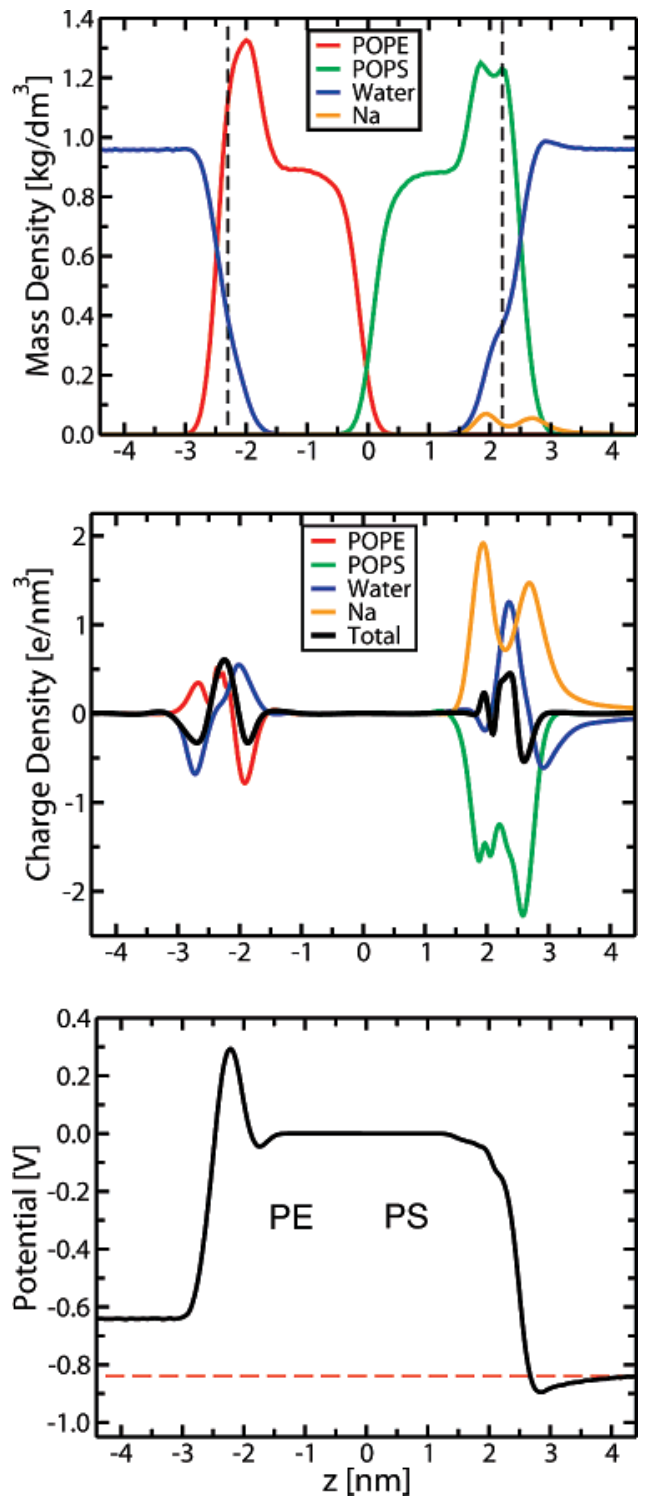

Figure 3. (top) Component-wise mass density profiles of an asymmetric POPS/POPE membrane versus distance $z$ from the center of mass of the membrane. (middle) Charge density profiles of a POPS/ POPE membrane. (bottom) Electrostatic potential across a POPS/POPE membrane. The potential is chosen to be zero at the center of mass of the membrane.

leaflet and of a mixed PC/PS leaflet, where the latter case contained around $33 \%$ of anionic lipids. ${ }^{48}$ However, as the authors employed a single bilayer setup, the counterions of PS lipids were able to interact with the purely zwitterionic leaflet, making the overall picture smeared. Furthermore, the method used for calculating the electrostatic potential did not allow the authors to get symmetric potential profiles even for symmetric PC bilayers, rendering a direct comparison with our results hardly possible.

C. Asymmetric PS/PE Membrane. To complement our findings, we performed additional atomic-scale MD simulations for another asymmetric zwitterionic/anionic membrane in which POPC was replaced by POPE (see Methods for details). The major difference between these zwitterionic lipids is that POPE lipids are more capable for hydrogen bonding. ${ }^{49}$ For the PS/PE bilayer, one hence expects a more densely packed structure that is indeed readily observable from the peak heights of the density profiles (see Figure 3 (top)).
Water permeates into the POPE leaflet to a significantly lesser extent as compared to the zwitterionic side of a PS/PC membrane (see Figures 2 and 3), reflecting the well-known difference in hydration level of PC and PE membranes. ${ }^{49}$ This results in an insufficient compensation of partial charges of the polar PE head group and in a rather sharp peak in the total charge density profile in the POPE leaflet, the peak being higher than that observed on the neutral side of a PS/PC membrane (see Figures 2 (middle) and 3 (middle), and also ref 10). As a consequence, the potential difference across the PE monolayer is larger than what was seen in a PC monolayer, ${ }^{10}$ and one can expect a somewhat smaller magnitude of the intrinsic potential across the entire asymmetric membrane. Indeed, the potential difference between the two surfaces of an asymmetric PS/PE membrane turns out to be nonzero and amounts to $198 \pm 8$ $\mathrm{mV}$, which is smaller than the value of $238 \mathrm{mV}$ observed for a PS/PC membrane.

On the experimental side, the electrostatic properties of an asymmetric PS/PE membrane has been probed by the nonactin$\mathrm{K}^{+}$complex. ${ }^{8}$ The experimentally observed shape of the electrostatic potential across the PS/PE membrane is very similar to what was observed in MD simulations, the potential on the anionic side being lower than that on the neutral (zwitterionic) side. The experimental value for the potential difference between the two sides of the membrane was found to be $86 \mathrm{mV} .{ }^{8}$ The value for the membrane potential measured through the simulations $(198 \mathrm{mV})$, while being of the same order of magnitude, turns out to be considerably larger than the experimental one. The difference between simulations and experiment is obviously in part due to error margins in both experiment and simulations, and also due to current limitations in force fields. However, we consider that the important issue here is the different conditions used: the experiment was performed at a lower temperature and in the presence of $\mathrm{KCl}$ salt. ${ }^{8}$ The role of salt is particularly relevant here, since the results in Figure 3 show that the charge density profile of counterions competes against the corresponding one arising from PS head groups. On these grounds, it is possible that the presence of salt decreases the total membrane potential; preliminary data is in favor of this view (unpublished data).

Nonetheless, one can conclude that the main findings of our atomic-scale MD simulations are in general agreement with available experimental data.

\section{Conclusions}

To explore how asymmetry in transmembrane distribution of charged (anionic) lipids across plasma membranes affects membrane electrostatics, we performed atomic-scale MD simulations of asymmetric membranes with one leaflet composed of zwitterionic PC or PE lipids, and another leaflet built from anionic PS lipids. Special care was taken to ensure that counterions of the anionic leaflet do not affect its zwitterionic counterpart. For doing that, a double bilayer setup (i.e., two lipid bilayers in a simulation box) was employed.

It turned out that both $\mathrm{PS} / \mathrm{PE}$ and $\mathrm{PS} / \mathrm{PC}$ asymmetric membranes are characterized by a nonzero potential difference between two membrane surfaces. This potential arises from the difference in the surface charges of the two leaflets of asymmetric PS/PC and PS/PE membranes. Remarkably, the intrinsic potential has a lower value on the anionic side of membranes, so that the potential due to transmembrane asymmetry is of the same sign as the membrane potential in cells. The magnitude of the intrinsic membrane potential was found to be $238 \mathrm{mV}$ for a PS/PC membrane and $198 \mathrm{mV}$ for a PS/PE membrane. Our findings, being in reasonable agreement with available 
experimental data, lend support to the idea that transmembrane lipid asymmetry contributes to the membrane potential of plasma membranes.

Further research in the area could lie in the development of more realistic membrane models that would match closely the lipid composition of cellular membranes. Of particular interest are asymmetric membranes with PC, sphingomyelin, and cholesterol in one leaflet and PE and PS in another. The presence of cholesterol, in particular, is of exceptional biological interest. ${ }^{50-52}$ Furthermore, as charged lipids such as cardiolipins are abundant in mitochondrial membranes, ${ }^{53}$ the role of membrane lipid composition on the electrostatics and membrane potential in these cellular power plants would deserve to be clarified. Finally, cationic liposomes ${ }^{54-57}$ are increasingly being considered for use in gene therapy, yet the role of lipid asymmetry on their electrostatic properties is only weakly understood.

Acknowledgment. The authors thank Peter Tieleman for providing the structure and topology files of a POPS bilayer. Funding from the Academy of Finland (I.V.) is gratefully acknowledged. The simulations were performed at the Finnish IT Center for Science and on the HorseShoe (DCSC) supercluster at the University of Southern Denmark.

\section{References and Notes}

(1) Gennis, R. B. Biomembranes: Molecular Structure and Function; Springer-Verlag: New York, 1989.

(2) Zachowski, A. Biochem. J. 1993, 294, 1-14.

(3) Manno, S.; Takakuwa, Y.; Mohandas, N. Proc. Natl. Acad. Sci. U.S.A. 2002, 99, 1943.

(4) Balasubramanian, K.; Schroit, A. J. Annu. Rev. Physiol. 2003, 65, $701-734$

(5) McLaughlin, S. Annu. Rev. Biophys. Biophys. Chem. 1989, 18, $113-136$.

(6) Voet, D.; Voet, J. G. Biochemistry, 3rd ed.; John Wiley \& Sons: New York, 2004.

(7) Latorre, R.; Hall, J. E. Nature 1976, 264, 361-363.

(8) Hall, J. E.; Latorre, R. Biophys. J. 1976, 15, 99-103.

(9) Wiese, A.; Reiners, J. O.; Brandenburg, K.; Kawahara, K.; Zahringer, U.; Seydel, U. Biophys. J. 1996, 70, 321-329.

(10) Gurtovenko, A. A.; Vattulainen, I. J. Am. Chem. Soc. 2007, 129, 5358-5359

(11) Schlegel, R. A.; Williamson, P. Cell Death Differ. 2001, 8, 551563.

(12) Böckmann, R. A.; Hac, A.; Heimburg, T.; Grubmüller, H. Biophys. J. 2003, 85, 1647-1655.

(13) Garcia-Manyes, S.; Oncins, G.; Sanz, F. Biophys. J. 2005, 89, 1812-1826.

(14) Garcia-Manyes, S.; Oncins, G.; Sanz, F. Electrocim. Acta 2006, 51, 5029-5036.

(15) Fukuma, T.; Higgins, M. J.; Jarvis, S. P. Phys. Rev. Lett. 2007, 98, 106101.

(16) Böckmann, R. A.; Grubmüller, H. Angew. Chem., Int. Ed. 2004, 43, 1021-1024.

(17) Pandit, S. A.; Bostick, D.; Berkowitz, M. L. Biophys. J. 2003, 84, $3743-3750$.

(18) Gurtovenko, A. A. J. Chem. Phys. 2005, 122, 244902.

(19) Gurtovenko, A. A.; Vattulainen, I. J. Phys. Chem. B 2008, 112, 1953-1962.

(20) Berger, O.; Edholm, O.; Jahnig, F. Biophys. J. 1997, 72, 20022013.

(21) Tieleman, D. P.; Berendsen, H. J. C. Biophys. J. 1998, 74, 27862801.
(22) Mukhopadhyay, P.; Monticelli, L.; Tieleman, D. P. Biophys. J. 2004, $86,1601-1609$

(23) Berendsen, H. J. C.; Postma, J. P. M.; van Gunsteren, W. F.; Hermans, J. In Intermolecular Forces; Pullman, B., Ed.; Reidel: Dordrecht, The Netherlands, 1981; pp 331-342.

(24) Lindahl, E.; Hess, B.; van der Spoel, D. J. Mol. Model. 2001, 7, $306-317$.

(25) Darden, T.; York, D.; Pedersen, L. J. Chem. Phys. 1993, 98, 1008910092

(26) Essman, U.; Perera, L.; Berkowitz, M. L.; Darden, T.; Lee, H.; Pedersen, L. G. J. Chem. Phys. 1995, 103, 8577-8592.

(27) Berendsen, H. J. C.; Postma, J. P. M.; van Gunsteren, W. F.; DiNola,

A.; Haak, J. R. J. Chem. Phys. 1984, 81, 3684-3690.

(28) Hyslop, P. A.; Morel, B.; Sauerheber, R. D. Biochemistry 1990 29, $1025-1038$

(29) Lantzsch, G.; Binder, H.; Heerklotz, H.; Wendling, M.; Klose, G. Biophys. Chem. 1996, 58, 289-302.

(30) König, B.; Dietrich, U.; Klose, G. Langmuir 1997, 13, 525-532.

(31) Smaby, J. M.; Momsen, M. M.; Brockman, H. L.; Brown, R. E. Biophys. J. 1997, 73, 1492-1505.

(32) Mukhopadhyay, P.; Vogel, H. J.; Tieleman, D. P. Biophys. J. 2004 $86,337-345$

(33) Leekumjorn, S.; Sum, A. K. J. Phys. Chem. B. 2007, 111, 60266033.

(34) de Vries, A. H.; Mark, A. E.; Marrink, S. J. J. Phys. Chem. B 2004, $108,2454-2463$.

(35) Cascales, J. J. L.; de la Torre, J. G.; Marrink, S. J.; Berendsen, H.

J. C. J. Chem. Phys. 1996, 104, 2713-2720.

(36) Pandit, S. A.; Berkowitz, M. L. Biophys. J. 2002, 82, 1818-1827.

(37) Rand, R. P.; Parsegian, V. A. Biochim. Biophys. Acta 1989, 988, $351-376$.

(38) MacDonald, R. C.; Simon, S. A.; Baer, E. Biochemistry 1976, 15, $885-891$.

(39) Cevc, G.; Watts, A.; Marsh, D. Biochemistry 1981, 20, 4955-4965.

(40) Sachs, J. N.; Crozier, P. S.; Woolf, T. B. J. Chem. Phys. 2004, $121,10847-10851$

(41) Gurtovenko, A. A.; Vattulainen, I. J. Am. Chem. Soc. 2005, 127, $17570-17571$.

(42) Gurtovenko, A. A.; Vattulainen, I. Biophys. J. 2007, 92, 18781890 .

(43) Kandasamy, S. K.; Larson, R. G. J. Chem. Phys. 2006, 125, 074901.

(44) Leekumjorn, S.; Sum, A. K. Biochim. Biophys. Acta 2006, 1758 , $1751-1758$

(45) Vernier, P. T.; Ziegler, M. J.; Sun, Y.; Gundersen, M. A.; Tieleman, D. P. Phys. Biol. 2006, 3, 233-247.

(46) Bostick, D.; Berkowitz, M. L. Biophys. J. 2003, 85, 97-107.

(47) Petrache, H. I.; Tristram-Nagle, S.; Gawrisch, K.; Harries, D.; Parsegian, V. A.; Nagle, J. F. Biophys. J. 2004, 86, 1574-1586.

(48) Cascales, J. J. L.; Otero, T. F.; Smith, B. D.; Gonzalez, C.; Marquez, M. J. Phys. Chem. B 2006, 110, 2358-2363.

(49) McIntosh, T. J. Chem. Phys. Lipids 1996, 81, 117-131.

(50) Aittoniemi, J.; Rog, T.; Niemela, P.; Pasenkiewicz-Gierula, M.; Karttunen, M.; Vattulainen, I. J. Phys. Chem. B 2006, 110, 25562-25564. (51) Aittoniemi, J.; Niemela, P. S.; Hyvonen, M. T.; Karttunen, M.; Vattulainen, I. Biophys. J. 2007, 92, 1125-1137.

(52) Niemela, P. S.; Ollila, S.; Hyvonen, M. T.; Karttunen, M.; Vattulainen, I. PLoS Comput. Biol. 2007, 3, 304-312.

(53) Lodish, H.; Berk, A.; Zipursky, S. L.; Matsudaira, P.; Baltimore, D.; Darnell, J. E. Molecular Cell Biology; W. H. Freeman and Company: New York, 2000.

(54) Pitard, B.; Aguerre, O.; Airiau, M.; Lachages, A. M.; Boukhnikachvili, T.; Byk, G.; Dubertret, C.; Herviou, C.; Scherman, D.; Mayaux, J. F.; Crouzet, J. Proc. Natl. Acad. Sci. U.S.A. 1997, 94, 14412-14417.

(55) Zantl, R.; Baicu, L.; Artzner, F.; Sprenger, I.; Rapp, G.; Rädler, J. O. J. Phys. Chem. B 1999, 103, 10300-10310.

(56) Gurtovenko, A. A.; Patra, M.; Karttunen, M.; Vattulainen, I. Biophys. J. 2004, 86, 3461-3472.

(57) Gurtovenko, A. A.; Miettinen, M.; Karttunen, M.; Vattulainen, I. J. Phys. Chem. B 2005, 109, 21126-21134.

(58) Thijsse, B. J.; Hollanders, M. A.; Hendrikse, J. Comput. Phys. 1998, $12,393-399$. 